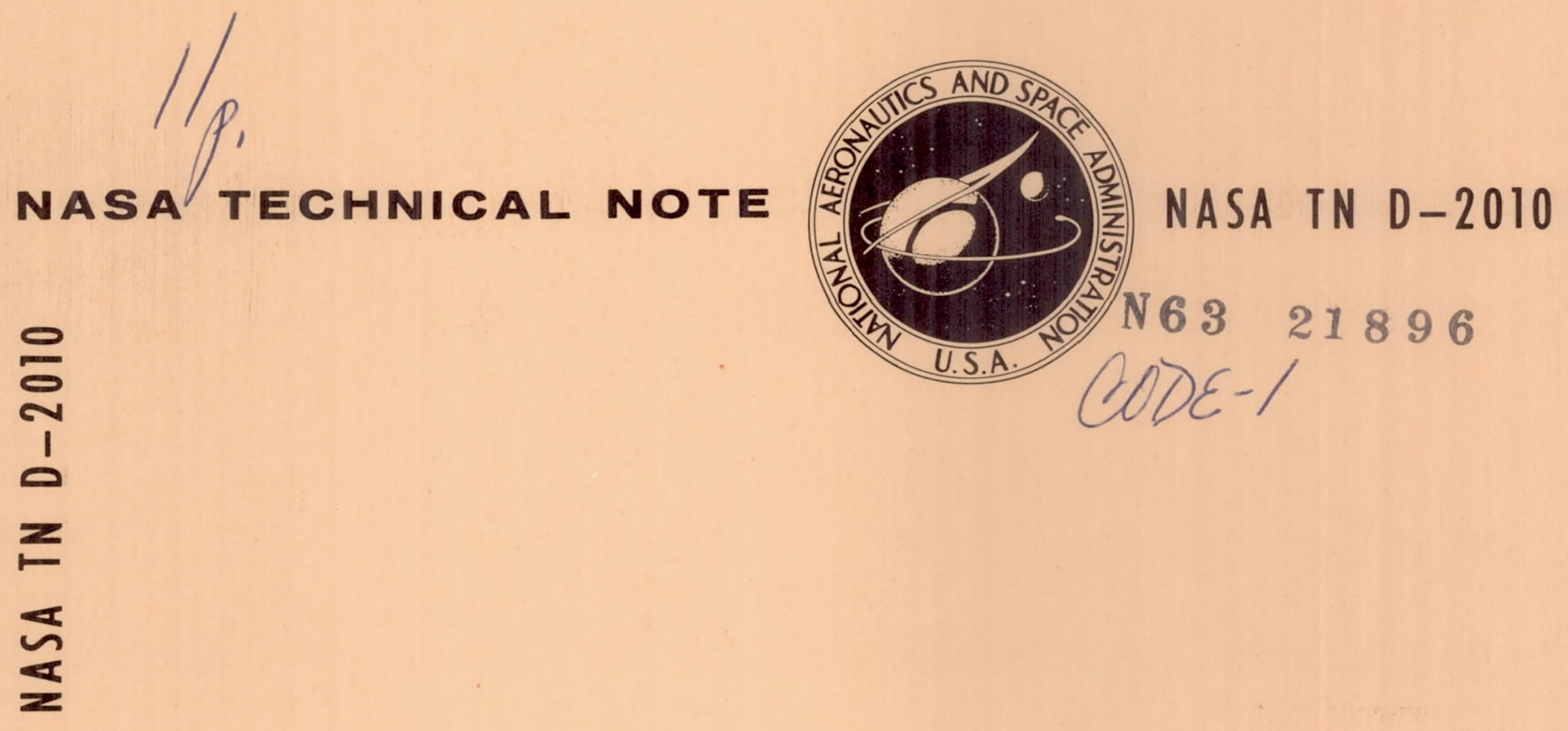

\title{
CALCULATION OF ELECTRON ENERGY DEPOSITION IN THIN-FILM POLYMERIC MATERIALS
}

by Louis A. Teichman and

Ernest S. Armstrong

Langley Research Center

Langley Station, Hampton, Va.

NATIONAL AERONAUTICS AND SPACE ADMINISTRATION - WASHINGTON, D.C. OCTOBER 1963 
TECHNICAL NOTE D-2010

\title{
CALCULATION OF ELECTRON ENERGY DEPOSITION \\ IN THIN-FILM POLYMERIC MATERIALS
}

\author{
By Louis A. Teichman and Ernest S. Armstrong \\ Langley Research Center \\ Langley Station, Hampton, Va.
}





\section{NATIONAL AERONAUTICS AND SPACE ADMINISTRATION \\ TECHNICAL NOTE D-2010 \\ CALCULATION OF ELECTRON ENERGY DEPOSITION IN THIN-FIIM \\ POLYMERIC MATERIALS}

By Louis A. Teichman and Ernest S. Armstrong

SUMMARY

This report deals with the analysis and computation of the energy deposited by electrons in shielded and unshielded polymeric films. The analysis employs numerical methods to determine the average path length of an electron in a material for which the atomic density, atomic number, and mean excitation potential are known. Consideration is limited to electrons with incident kinetic energies up to $5 \mathrm{Mev}$ which strike the material normal to its surface.

\section{INTRODUCTION}

With the ever-increasing use of polymers in the exploration and utilization of space, as exemplified by the Echo I and Explorer IX inflatable satellites, it is necessary to analyze the effect of the space environment on such materials. High-energy electrons, such as those found in the Van Allen belts, are an important part of this environment. The purpose of the present paper is to indicate a numerical approach to computing the energy deposited by electrons in thin polymer films, such as those employed in erectable space structures. Curves and equations are presented for use in computing energy deposition in these films. Some computed results are given for energy deposited as a function of incident energy, for electrons incident normally on aluminum-shielded and unshielded polypropylene and Mylar.

\section{SYMBOLS}

a.

$-d E / d x \quad$ rate of loss of kinetic energy per unit path length, erg/cm

e electronic charge, statcoulomb

$E$

I ratio of actual path length to range

electron kinetic energy, erg

mean excitation energy, erg 


$\begin{array}{ll}\mathrm{m} & \text { electronic rest mass, gram } \\ \mathrm{N} & \text { average number of atoms per unit volume (atomic density), } \mathrm{cm}^{-3} \\ \mathrm{v} & \text { instantaneous electron velocity, } \mathrm{cm} / \mathrm{sec} \\ \mathrm{X} & \text { average electron-path length, } \mathrm{cm} \\ \mathrm{x} & \text { distance along path, } \mathrm{cm} \\ \mathrm{y} & \text { material thickness, cm } \\ \mathrm{Z} & \text { mean atomic number } \\ \beta & \text { ratio of electron velocity to velocity of light in vacuum } \\ \text { Subscripts: } & \text { incident condition } \\ 0 & \text { exit condition } \\ \mathrm{l}\end{array}$

THEORY

As an electron passes through matter, it loses energy as a result of ionization, excitation, and radiation. In these phenomena, the electron suffers deflections, caused by a series of individual random scattering processes, such that it typically follows a tortuous path. Hence, when it passes through a thin film, the path length within the film is considerably greater than if it had proceeded through the film in a straight line; and there is a correspondingly greater energy deposition in the film. It has been empirically determined (ref. I), through cloud chamber and photoemulsion pack studies, that the ratio a of path length to range varies from 1.2 to 4, depending on the energy of the electron and the atomic composition of the material (ref. 2). The lower value 1.2 applies to high-energy electrons, that is, above a few $\mathrm{Kev}$, penetrating materials of low atomic number. For the polymer films and the electron energies considered in this report, this lower value is the more applicable.

The increase in path length of electrons penetrating a thin film over that of a straight line also results in large variations in the energy lost by electrons of the same incident energy. It is, however, extremely difficult to consider the problem of electron energy deposition on a statistical basis in which the instantaneous energy losses, path lengths, and scattering angles are used. Therefore, with the realization that inaccuracies of the order of perhaps 50 percent or more at the lower energies are involved, use has been made of a specific average energy loss and path length for all electrons with the same incident energy, the ratio a has been held fixed at 1.2 for all the examples cited, and consideration has been limited to electrons entering the material normal to its surface. In any case, the accuracy involved in making these assumptions is 
probably greater than that with which the flux of electrons in the earth's radiation zone is known.

If the foregoing assumptions are made, the average path length $X$ of an electron penetrating a material is given by

$$
X=\int_{0}^{E} \frac{d E}{-d E / d x}
$$

where $E$ represents the kinetic energy of an incident electron and $-\mathrm{dE} / \mathrm{dx}$ represents the rate at which kinetic energy is being lost per unit path length. This quantity is given by (ref. 3):

$$
\begin{aligned}
\frac{-\partial E}{d x}= & \frac{2 \pi N e^{4} z}{m v^{2}}\left[\log _{e} \frac{m v^{2} E}{2 I^{2}\left(1-\beta^{2}\right)}-\left(\log _{e} 2\right)\left(2 \sqrt{1-\beta^{2}}-1+\beta^{2}\right)+1-\beta^{2}\right. \\
& \left.+\frac{1}{8}\left(1-\sqrt{1-\beta^{2}}\right)^{2}\right]
\end{aligned}
$$

The mean excitation energy is approximated by setting $I=13.6 \mathrm{z}$ ( $r e f$. 4), where the constant 13.6 represents the ionization potential of the hydrogen atom in electron volts.

With the substitution of equation (2), equation (1) may be integrated numerically. From the results may be constructed a plot of the logarithm of the average path length against the logarithm of the incident kinetic energy. The resultant curve is a parabola fitting the generalized expression

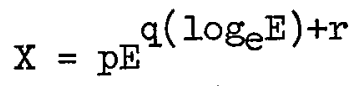

where $p, q$, and $r$ are parameters of the material being studied.

By using equation (3), the total average path $X$ of an electron with any incident kinetic energy $E$ may be found. In order to determine the exit energy of an electron whose range is greater than the material thickness $y$ and thereby the amount of energy absorbed, the following procedure is used:

(1) The actual distance traveled by the electron in the material ay is subtracted from the distance $x_{0}$ that the electron would have to travel in the material before it lost all its energy. 
(2) The new quantity $x_{1}=x_{0}-a y$ is the path length of an electron with an exit energy $E_{1}$. The exit energy is determined by substituting $X_{I}$ into equation (3).

(3) To determine the energy deposited, subtract the exit energy $E_{1}$ from the incident energy $\mathrm{E}_{\mathrm{O}}$.

(4) In order to determine the amount of energy deposited in a shielded material, the energy of an electron after it has traversed the outer shielding material is used as the energy which is incident on the material being shielded. Repeated application of this method will yield the amount of electron energy deposited in any or all layers of a multiwalled structure.

In theory, this approach is not restricted to electrons that are incident normal to the surface - one need merely correct each penetration distance, or film thickness, by the secant of the incidence angle (as measured from the normal). Because of the previously mentioned tortuosity of the path, however, the simple approach becomes more questionable for the higher angles of incidence; nevertheless, it would probably be adequate for estimating the deposited energy in an omnidirectional electron field.

\section{RESULTS}

In the present examples, equation (1) was solved numerically by using Simpson's one-third rule (ref. 5), with increments of kinetic energy equal to $0.001 \mathrm{Mev}$ below $2 \mathrm{Mev}$ and $0.01 \mathrm{Mev}$ above $2 \mathrm{Mev}$, for polypropylene, Mylar, and aluminum. The resultant parabolas are shown in figure 1. The following equations (compare eq. (3)) were fitted to the parabolas:

Aluminum:

$$
X=154 \mathrm{E}^{-0.0929 \log _{e} E+1.23}
$$

Polypropylene:

$$
X=256 E^{-0.0952 \log _{e} E+1.26}
$$

Mylar:

$$
X=301 E^{-0.0955 \log _{e} E+1.25}
$$

In these three equations, $X$ is expressed in mils ( 0.001 inch), and $\mathrm{E}$ is expressed in Mev and varies from 0.010 to $5.00 \mathrm{Mev}$. Beyond $0.040 \mathrm{Mev}$, the exror in fitting the curves to these expressions is less than 2 percent.

Using these expressions and the procedure previously outlined, values of energy deposited against incident kinetic energy, for normal incidence, were 


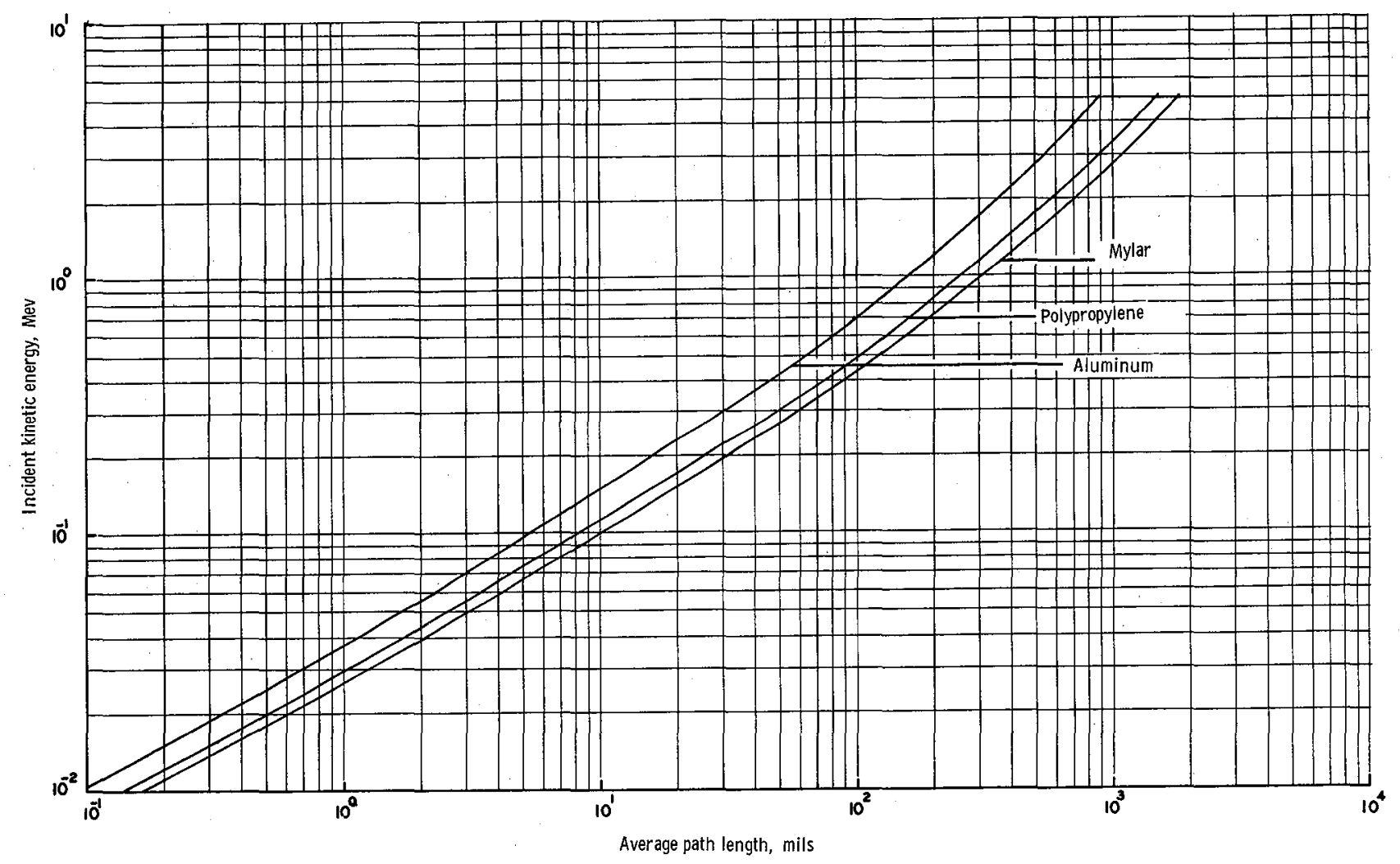

Figure 1.- Average path length as a function of incident electron kinetic energy for aluminum, Mylar, and polypropylene.

calculated for the following systems, with the ratio a of path length to range equal to 1.2:

(1) A I-mil-thick sheet of polypropylene.

(2) A 1-mil-thick sheet of polypropylene shielded by 1 mil of aluminum foil.

(3) The Echo II satellite laminate.

These curves are presented in figures 2, 3, and 4, respectively.

Since, in light materials, bremsstrahlung only becomes significant at about $10 \mathrm{Mev}$ (ref. 6), the predominant mode of energy loss is through the processes of ionization and excitation. Therefore, in the cases considered herein, essentially all of the energy lost by an electron is absorbed by the material being penetrated. 
In figure 2, the curve for l-mil polypropylene is linear in the region between the incident kinetic energies of 0 and $0.032 \mathrm{Mev}$. This linearity is due to the fact that an electron with incident energy below $0.032 \mathrm{Mev}$ has a range which is less than the film thickness. The electron will therefore remain in the film. Above $0.032 \mathrm{Mev}$, the curve decreases, reaches a minimum at about $1.65 \mathrm{Mev}$, and then rises slowly thereafter. The region from 0.032 to $5.00 \mathrm{Mev}$ therefore reflects the typical loss of electron energy in matter.

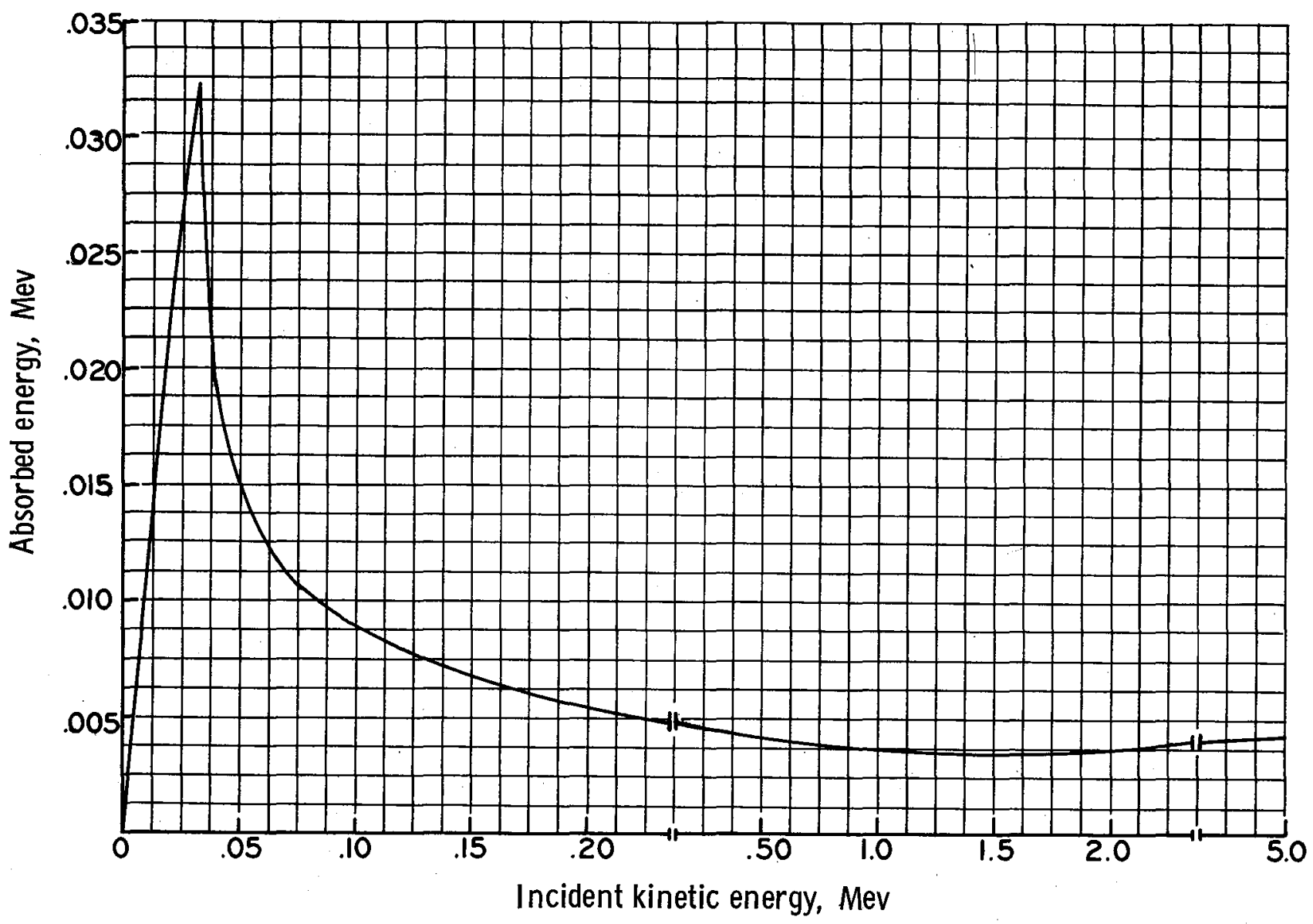

Figure 2.- Energy absorbed as a function of incident electron kinetic energy for l-mil unshielded polypropylene.

The curve in figure 3 follows the same general pattern as that in figure 2. It is, however, shifted to the right and the peak is somewhat narrower. Both of these differences are due to the presence of 1 mil of aluminum protecting the polypropylene. For incident energies below $0.040 \mathrm{Mev}$, the energy deposited in the polypropylene is zero, since all electrons are stopped in the aluminum. Between 0.040 and $0.055 \mathrm{Mev}$, the curve rises sharply but is not linear. This section of the curve represents electrons with energy sufficient to traverse the 
aluminum shield but not the polypropylene underlayer. The lack of linearity is due to the fact that electrons with differing incident energies are slowed down in disproportionate amounts by the aluminum. Above $0.055 \mathrm{Mev}$, the curve decreases, reaches a minimum at about $1.65 \mathrm{Mev}$, and then rises slowly thereafter.

For the Echo II calculations, the satellite was considered to be inflated in space in the form of a perfect sphere with an evacuated interior. The shell consists of 0.35-mil Mylar sandwiched between two layers of 0.18-mil soft aluminum foil. Electrons emerging from one hemisphere are assumed to lose no energy in their passage to the other hemisphere. This system may consequently be considered as a five-layer laminate consisting of 0.18 -mil aluminum, 0.35-mil Mylar, $0.36-m i l$ aluminum, 0.35-mil Mylar, and 0.18-mil aluminum with electrons entering and emerging from both the upper and lower surfaces since the flux is assumed to be omnidirectional. Therefore, for electrons of energy sufficient to penetrate both layers of Mylar, the net amount of energy deposited in either layer is the sum of the energies deposited in both layers by an electron entering either surface.

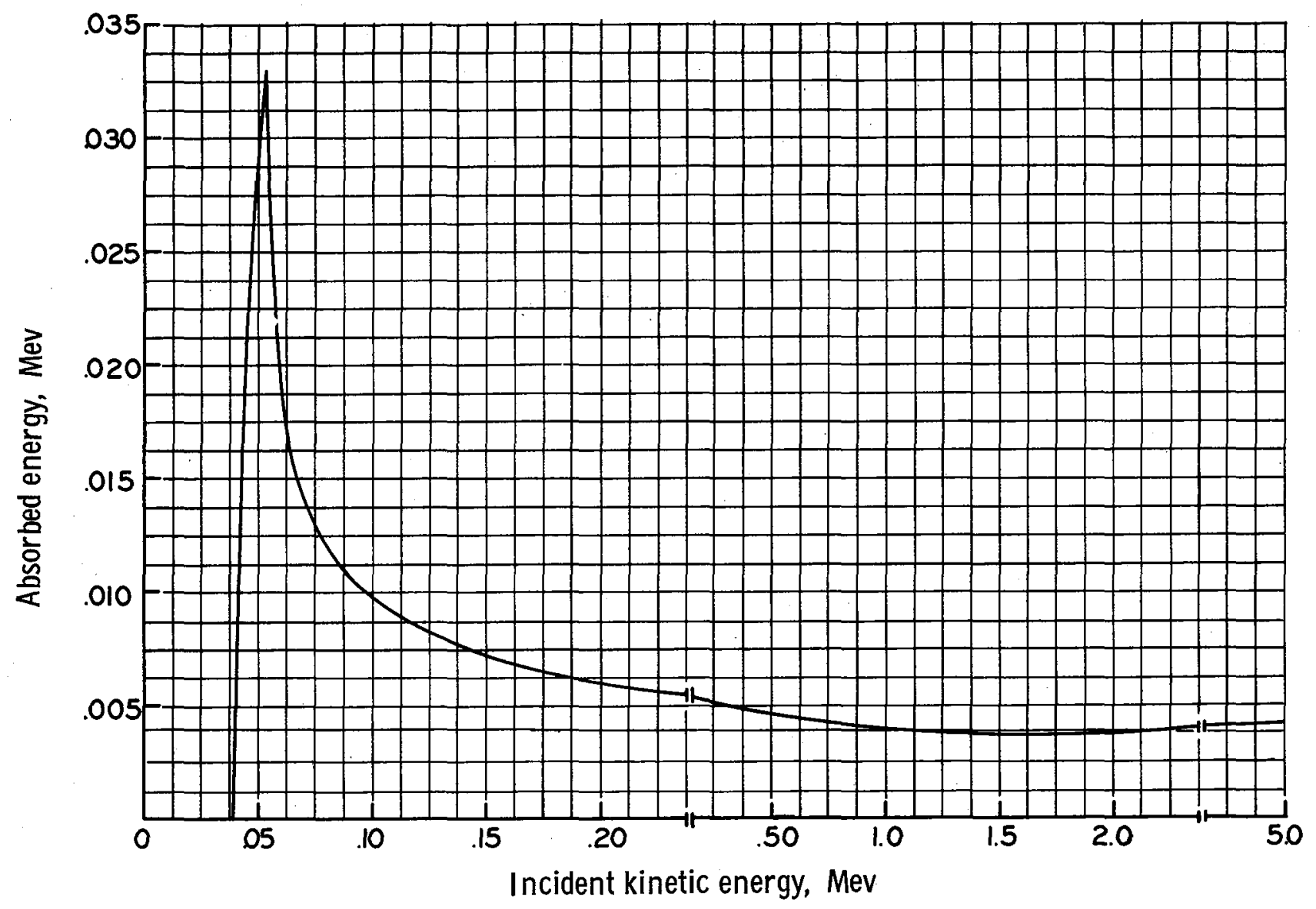

Figure 3.- Energy absorbed as a function of incident electron kinetic energy for 1-mil polypropylene shielded by l-mil aluminum. 
The curve of figure 4 is the result of this addition and shows two peaks. An electron with incident kinetic energy between 0 and $0.017 \mathrm{Mev}$ is stopped in the outer layer of aluminum and therefore deposits no energy in the Mylar. Electrons with energies between 0.017 and $0.025 \mathrm{Mev}$ are stopped in the first Mylar layer and produce a sharply rising nonlinear curve. Between 0.025 and $0.035 \mathrm{Mev}$, electrons completely penetrate the first Mylar layer and the typical decreasing curve results. The curve again rises sharply but nonlinearly between 0.035 and $0.040 \mathrm{Mev}$ because electrons with these energies are stopped in the second Mylar layer. The second decreasing region of the curve corresponds to electrons with sufficient energy to traverse completely the second. Mylar layer.

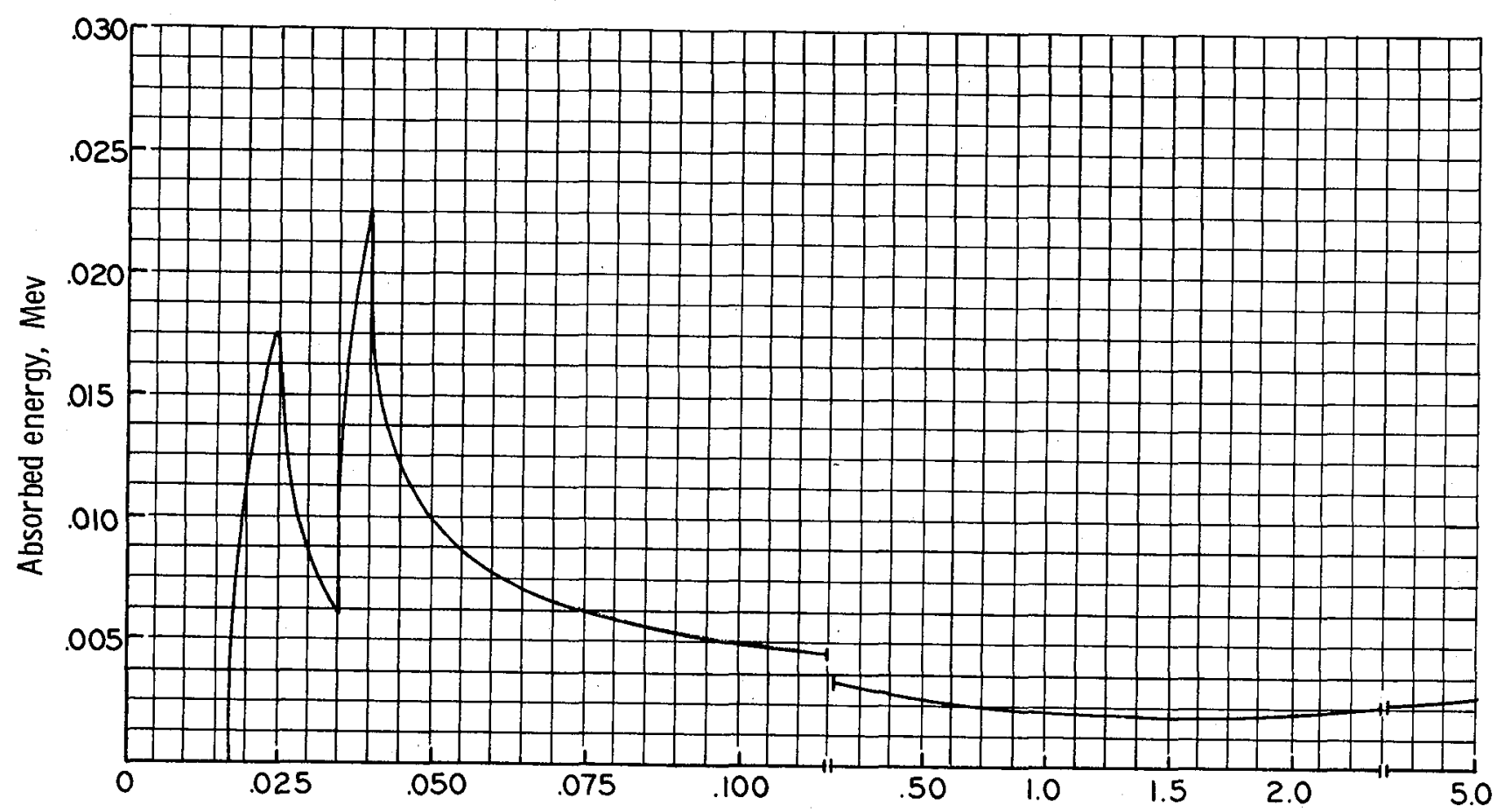

Incident kinetic energy, Mev

Figure 4.- Energy absorbed as a function of incident electron kinetic energy for the Mylar layers of the Echo II satelite laminate.

\section{CONCLUDING REMARKS}

A procedure has been presented for approximating the energy deposited in shielded and unshielded polymer films by electrons entering the material normal to the surface. It has been applied to three cases, including that of the 
inflated Echo II balloon, with incident electron kinetic energies up to 5 Mev. Characteristic variations of the deposited energy with incident electron kinetic energy have been derived.

\section{Langley Research Center,}

National Aeronautics and Space Administration, Langley Station, Hampton, Va., July 10, 1963.

REF'ERENCES

1. Williams, E. J.: The Rate of Loss of Energy by $\beta$-Particles in Passing Through Matter. Proc. Roy. Soc. (London), Ser. A, vol. 130, 1931, pp. 310-327.

2. Evans, Robley D.: The Atomic Nucleus. McGraw-Hill Book Co., Inc., c.1955.

3. Bethe, Hans A., and Ashkin, Julius: Passage of Radiations Through Matter. Experimental Nuclear Physics, Vol. I, Pt. II, Sec. 2, E. Segré, ed., John Wiley \& Sons, Inc., c.1953.

4. Nelms, Ann T.: Energy Loss and Range of Electrons and Positrons. NBS Circular 577, U.S. Dept. Commerce, July 1956.

5. Hildebrand, F. B.: Introduction to Numerical Analysis. McGraw-Hill Book Co., Inc., 1956.

6. Richtmyer, F. K., and Kennard, E. H.: Introduction to Modern Physics. Fourth ed., McGraw-Hill Book Co., Inc., c.1947. 